\title{
In situ synthesis of silver nanoparticles on the surface of PDMS with high antibacterial activity and biosafety toward an implantable medical device
}

\author{
Joong Hyun Kim, HyeungWoo Park and Soo Won Seo*
}

\begin{abstract}
We developed a straightforward method to fabricate antibacterial silicon films via the in situ synthesis of silver nanoparticles (AgNPs) on a polydimethylsiloxane (PDMS) film. To grow AgNPs attached on the film, AgNP seeds were synthesized through the reduction of silver ions electrostatically bound to hydroxyl groups formed on the surface of the film after treatment with air plasma. In the growth reaction, silver ions were reduced on the seeds of AgNPs by sodium citrate in a solution of $\mathrm{AgNO}_{3}$, which allowed for the formation of AgNPs with sizes of up to $\sim 500 \mathrm{~nm}$, which The formed AgNPs on the films were characterized using UV-vis spectrophotometer, scattering electron microscope and induced coupled mass spectrometer. The amount of AgNPs was estimated to be less than $0.05 \%$ of the total film weight. Even though it was coated with a small amount of AgNPs, the PDMS film exhibited reduction of $E$. coli and S. aureus with values of $\log _{10} 4.8$ and $\log _{10} 5.7$, respectively. The biosafety of the AgNP-attached PDMS film was examined by contact of cells with the film or film eluent. Counting of viable cells revealed no significant cytotoxicity of the in situ-fabricated AgNPs on the PDMS film.
\end{abstract}

Keywords: Antibacterial medical device, Silver nanoparticles, Silicone, Catheter, Oxygen plasma

\section{Introduction}

Since the first use of a silicone elastomer tube for duct repair in biliary surgery 60 years ago, silicone materials have been widely used in medical applications because of their superior biocompatibility compared with that of other materials [1]. Notably, PDMS-based elastomers satisfy the criteria for biocompatibility, including nonirritating and nonsensitizing behaviors. Additionally, PDMS is thermally and chemically stable. These characteristics have allowed for the long-term use of PDMS-based implants [2, 3]. However, the hydrophobic nature of PDMS results in the adhesion of bacteria on the surface of these devices, leading to microbial infections. In the United States, more than 250,000 primary blood stream infections occur annually among 150

\footnotetext{
*Correspondence: soowon@dgmif.re.kr

Medical Device Development Center, Daegu-Gyeongbuk Medical Innovation Foundation, Daegu 701-310, South Korea
}

million intravascular devices [4]. Antibacterial properties of medical devices can be achieved by coating the surface or mixing a PDMS elastomer with biocides, such as antibiotics, silver ion, furanones, or 3-(trimethoxysilyl)ropyldimethyloctadecylammonium chloride [5]. Among reported bacterial killing agents, silver nanoparticles (AgNPs) are attractive because they show unique properties such as long-term stability and broad antibacterial activities [6]. AgNP_coated implantable medical devices such as catheters, prosthetics, bone adhesives, contact lenses, and ureteral stents have shown promising results that support the use of AgNPs as potential antibacterial agents in such devices [7-10]. Several methods to fabricate polymer-nanoparticle composites have been reported, such as mixing AgNPs with elastomers [11], immobilization of AgNPs on the surface of polymers [12], and synthesizing AgNPs inside of elastomers [13]. The silver ions released from AgNPs play key roles in such systems demonstrating antibacterial effects. 
Since most AgNPs mixed with elastomers should not be exposed to the external environment, a small number of mixed AgNPs were used as antibacterial agents. Therefore, immobilizing methods are more effective than mixing methods. However, immobilizing methods are more complex and expensive than mixing methods because of the additional conjugating or layer-by-layer chemistry between the surface of polymers and AgNPs [11, 12]. Herein, we present a method to synthesize AgNPs on the surface of PDMS. An easy and facile process for the in situ fabrication of nanoparticles with no further conjugating chemistry for the polymer or nanoparticle is reported. Effective antibacterial activity of the nanocomposites against both $E$. coli and S. aureus was achieved. Additionally, no cellular toxicity of the nanocomposites was observed.

\section{Experimental}

Silver nitrate $\left(\mathrm{AgNO}_{3}, 99 \%\right)$, sodium hydroxide, hydrochloric acid, sodium chloride, yeast extract, D-glucose, meat extract, Mueller-Hinton broth (MHB), 3-(4,5-dimethylthiazol-2-yl)-2,5-diphenyltetrazoliumbromide (MTT), and sodium citrate (99\%) were purchased from Sigma-Aldrich. Polydimethylsiloxane (MED664) was purchased from Nusil. Acetone, isopropanol, and ethanol were obtained from Merck. Peptone, beef extract agar powder, Eagle's minimum essential medium (EMEM), and tryptone were purchased form BD Difco. Fetal bovine serum (FBS) was purchased from Fisher Scientific. For antibacterial tests, E. coli (ATCC 8739) and S. aureus (ATCC 6538P) were used. Cytotoxicity effects mediated by the PDMS film were tested using fibroblast cells.

The PDMS film was fabricated according to the manufacturer's instructions. In brief, part A and B of the kit were mixed thoroughly at a ratio of $1: 1$ by weight using an up-stirrer (IKA), and the mixture was then degassed under vacuum to remove any entrapped air bubble. The homogeneous mixture was uniformly dispersed on a Teflon film coated plate, and then was cured at $60{ }^{\circ} \mathrm{C}$ for 16-18 $\mathrm{h}$ under vacuum. The pre-cured PDMS film was cured at $120{ }^{\circ} \mathrm{C}$ for $1 \mathrm{~h}$ followed by additional curing at $150^{\circ} \mathrm{C}$ for another $1 \mathrm{~h}$. Unreacted elastomers and solvent were removed by successive rounds of ultrasonic cleaning of the cured film in acetone, isopropanol and distilled water, respectively, for $30 \mathrm{~min}$ per wash. This cleaning step was repeated two more times.

To synthesize AgNPs on the the PDMS films, the films underwent air plasma treatment in a $18 \mathrm{~W}$ plasma cleaner (PDC-32G-2, Harrick Plasma) for 10 min under vacuum. To form Ag seeds on the film, immediately after the plasma treatment the PDMS films were immersed in $150 \mathrm{ml} \mathrm{AgNO} 3(10 \mathrm{mM})$ solution at room temperature for $1 \mathrm{~h}$, followed by the addition of $3 \mathrm{ml} \mathrm{NaBH} / 40 \mathrm{mM})$ solution and incubation for 15 min. To grow $\mathrm{Ag}$ seeds, the PDMS film in $150 \mathrm{ml} 0.0167 \% \mathrm{AgNO}_{3}$ was heated to $80{ }^{\circ} \mathrm{C}$, followed by the injection of $3 \mathrm{ml} \mathrm{1 \%}$ (by weight) sodium citrate (Sigma-Aldrich) as a reducing agent of silver ions. After injecting sodium citrate, the color of the solution that contained the PDMS film turned to brown within $5 \mathrm{~min}$. The AgNP growth reaction was incubated for $2 \mathrm{~h}$ and repeated once or twice as needed. Residues on the film, such as the organic solvent from the elastomers, were removed by ultrasonic cleaning, as described above. The formation of AgNPs on the PDMS films was monitored by measuring the absorbance spectra of the film with a UV-Vis-NIR spectrometer (Agilent Technologies, Cary 5000). The size and shape of the AgNPs were investigated by imaging the films using a scattering emission microscope (Hitachi SU8010). The amount of AgNPs on the film was analyzed with an inductively coupled plasma mass spectrometer (Agilent ICP-MS 7700S).

To determine the optimal number of rounds of the AgNP growth reaction, antibacterial activity was determined by spraying $E$. coli cultures on a PDMS film and measuring the numbers of colonies on the film [14]. First, a $100 \mathrm{ml}$ aliquot of a bacterial suspension in PBS $\left(\sim 10^{11}\right.$ cells per $\left.\mathrm{ml}\right)$ that had been stored at $-20{ }^{\circ} \mathrm{C}$ was thawed at room temperature. The bacterial suspension was added to a sterile $50 \mathrm{ml}$ centrifuge tube that contained $20 \mathrm{ml}$ yeast dextrose broth and was shaken at $200 \mathrm{rpm}$ at $20{ }^{\circ} \mathrm{C}$ overnight. The next day, the bacterial suspension was centrifuged at $6000 \mathrm{rpm}(11,470 \times g)$ at room temperature for $10 \mathrm{~min}$, and the bacterial pellet was resuspended in $20 \mathrm{ml}$ PBS and vortexed for $2 \mathrm{~min}$; this step was repeated once more. The harvested bacterial cells were diluted in PBS to a final concentration of $5 \times 10^{7}$ cells per $\mathrm{ml}$ by measuring the OD of the bacterial suspension at $540 \mathrm{~nm}$ (an OD value of $\sim 1$ indicates $10^{9}$ bacterial cells per $\mathrm{ml}$ ). The diluted bacterial suspensions were sprayed onto a PDMS film at a rate of $\sim 10 \mathrm{ml} \mathrm{min}{ }^{-1}$ by adjusting the air flow with a chromatography sprayer. Films were air dried for $2 \mathrm{~min}$ at room temperature and placed in a Petri dish covered with solid growth agar $\left(25 \times 75 \mathrm{~mm}^{2}\right)$ followed by incubation of the Petri dish after sealing with Parafilm at $37^{\circ} \mathrm{C}$ overnight. Colonies that had grown on the tested film were then compared. The reduction in bacterial growth by the AgNP-grown PDMS film was estimated according to a modified version of ISO 22196, a protocol for measuring the antimicrobial activity of plastic surfaces [15]. A total of $10 \mathrm{ml} \mathrm{MHB}$ was inoculated and cultured overnight at $37^{\circ} \mathrm{C}$ in a shaker. On the day of the test, $200 \mu \mathrm{l}$ of the overnight culture was grown in $10 \mathrm{ml}$ fresh $\mathrm{MHB}$ and shaken at $37^{\circ} \mathrm{C}$ for $1 \mathrm{~h}$ to achieve exponential growth. The $1 \mathrm{~h}$ culture was diluted with saline 
using a spectrophotometer to achieve a cell density of $10^{7}$ colony forming units (CFU) $\mathrm{ml}^{-1}$. Each film surface was inoculated with $50 \mu \mathrm{l} 10^{7} \mathrm{CFU} \mathrm{m}{ }^{-1}$ culture and covered with a polyethylene film to prevent drying of the inoculated culture. Each film was incubated at room temperature in a Petri dish sealed with Parafilm containing pieces of paper saturated with water to avoid drying of the test surface. Bacterial growth after $24 \mathrm{~h}$ of inoculation was determined by transferring the film to a $50-\mathrm{ml}$ conical tube filled with $10 \mathrm{ml} 0.9 \%$ sodium chloride solution, which was stirred for $30 \mathrm{~s}$ to recover the inoculum. Finally, $1 \mathrm{ml}$ of the resulting solution was serially diluted, plated on Mueller-Hinton agar, and incubated at $37{ }^{\circ} \mathrm{C}$ for $24 \mathrm{~h}$ to determine the final CFU. The $\log _{10}$ reduction was calculated from the initial inoculum by subtracting the $\log _{10}$ count from the control assay on the test surface.

Biological safety of the AgNP-fabricated on PDMS film was examined with the MTT assay using the film elute and direct contact of the film to the tested cells (fibroblast cells). The MTT assay was carried out according to ISO 1093-5 [16]. The PDMS film elute was prepared by immersing the PDMS film $\left(25 \times 75 \mathrm{~mm}^{2}\right)$ in $20 \mathrm{ml}$ EMEM that contained 10\% FBS and $1 \times$ antibiotic. Cells were exposed to the material elute or allowed to contact sterilized PDMS film $\left(10 \times 10 \mathrm{~mm}^{2}\right)$ for $24 \mathrm{~h}$. For the control group, $10^{5}$ cells were cultured for $24 \mathrm{~h}$, and then were cultured in fresh EMEM without serum for another $24 \mathrm{~h}$. Cell viability was determined using the MTT assay. The MTT solution was prepared at $1 \mathrm{mg} \mathrm{ml}^{-1}$ in complete medium immediately prior to use. Then, $0.6 \mathrm{ml}$ MTT solution was added to each well, and cells were incubated in a humidified atmosphere of $5 \% \mathrm{CO}_{2}$ at $37{ }^{\circ} \mathrm{C}$ for $4 \mathrm{~h}$. After the incubation period, supernatant was removed and dark blue formazan crystals that had formed were dissolved in $0.6 \mathrm{ml}$ ethanol. Plates were shaken for $5 \mathrm{~min}$ and then the blue solution was transferred to a 96-well plate; optical densities were read at $570 \mathrm{~nm}$ in a multiwell spectrophotometer (TECAN Infinite ${ }^{\circledR} 200$ ).

\section{Results and discussion}

The process used in the in situ synthesis of AgNPs on PDMS surfaces is illustrated in Fig. 1. AgNPs are easily formed by the reduction of silver ions with reducing agents (e.g., $\mathrm{NaBH}_{4}$, hydroquinone, or sodium citrate) [17]. To form AgNPs attached the polymer surface instead of in a solution, we introduced hydroxyl groups on the surface because this functional group has been used as anchors for the adsorption of positive metal ions $[18,19]$ and can be easily formed by exposure to an air plasma [20,21]. Figure 2 shows the spectra of the polymer films with different plasma exposure time for reactions with $\mathrm{NaBH}_{4}$ to form AgNPs on the surface. AgNPs, ranging from 10 to $40 \mathrm{~nm}$, have a typical plasmonic
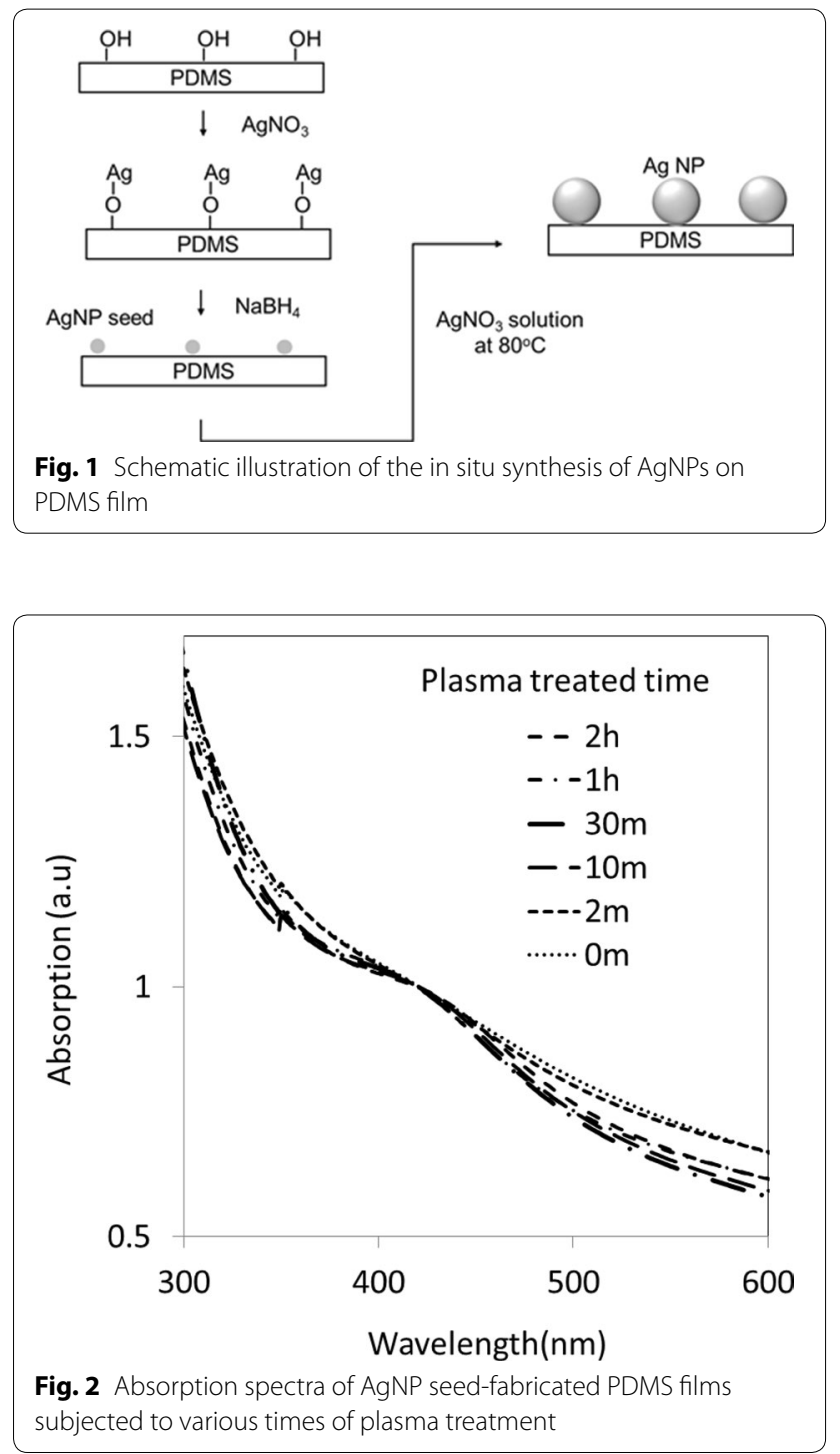

absorption spectrum with a maximum peak around $400 \mathrm{~nm}$. As shown in Fig. 2, the polymer films exposed to plasma for more than or equal to $10 \mathrm{~min}$ have maximum absorption peaks around $400 \mathrm{~nm}$, which indicates the formation of AgNPs. However, no difference of absorption spectrum was observed between the control film and film treated with plasma for less than $10 \mathrm{~min}$. This analysis of the absorption spectrum supports the role of hydroxyl group in capturing silver ions for the in situ formation of AgNPs. Since those PDMS films exposed to plasma for more than 10 min show no significant changes of the absorption, we used the PDMS films exposed to the air plasma for $10 \mathrm{~min}$ for further investigations.

In this study, all the PDMS films were fabricated with elastomers that contained xylene. Therefore, we recognized a need to investigate any growth inhibition of bacteria by residues of organic solvents for the elastomers 
on the polymer film. To remove xylene residues from the polymer film, we cleaned the PDMS film according to the manufacturer's protocol with successive washes of acetone and ethanol. Any growth inhibition of bacteria attributed to the organic solvent was estimated by comparing the number of colonies formed on the solventcleaned films and uncleaned control film at $24 \mathrm{~h}$ after spraying E. coli cultures on the films. Figure 3a, b show that more colonies were formed on the controls than on the solvent-cleaned film. This result indicates that the organic solvent residue could interfere with AgNPs in assessments of the inhibition of bacterial growth. Furthermore, the organic solvent is potentially hazardous for clinical applications. However, we removed the xylene residue on the polymer film for all tests of antibacterial activity and biosafety. We estimated the antibacterial activity of the $\mathrm{AgNO}_{3}$-reduced PDMS films by comparing the number of colonies formed on the film with that on a control film that was prepared without the $\mathrm{AgNO}_{2}$ reducing step. As shown in Fig. 3c, d, fewer bacterial colonies were observed on the $\mathrm{AgNO}_{3}$-reduced films $(\sim 40$ colonies) than on the control films ( $\sim 90$ colonies).
Notably, the FDA requires at least a four $\log _{10}$ reduction of bacteria for medical devices to be classified as showing antibacterial activity. Since we inferred that the amount of AgNPs on the film was not sufficient to adequately inhibit the growth of $E$. coli, additional growth reaction of AgNPs on the formed film was carried out. Figure 4 shows time-resolved images of the process used to promote the additional growth of AgNPs on the PDMS films in a solution that contained $\mathrm{AgNO}_{3}$ and sodium citrate as reducing agents. After $10 \mathrm{~min}$, the color of the $\mathrm{AgNO}_{3}$-containing solution clearly turned to brown, which indicated the formation of AgNPs in the solution. As the reaction time proceeded, the color of the solution became darker (Fig. 4). The growth of AgNPs on the polymer films was investigated by measuring absorption spectra and obtaining electron microscopic images of the polymer films. Figure 5 shows the absorption spectra of PDMS films with a typical plasmonic absorption spectrum of AgNPs after the additional growth of AgNPs. Compared with the results presented in Fig. 1, the significantly increased plasmonic absorption peak of AgNPs indicates that an increased portion of AgNPs was present
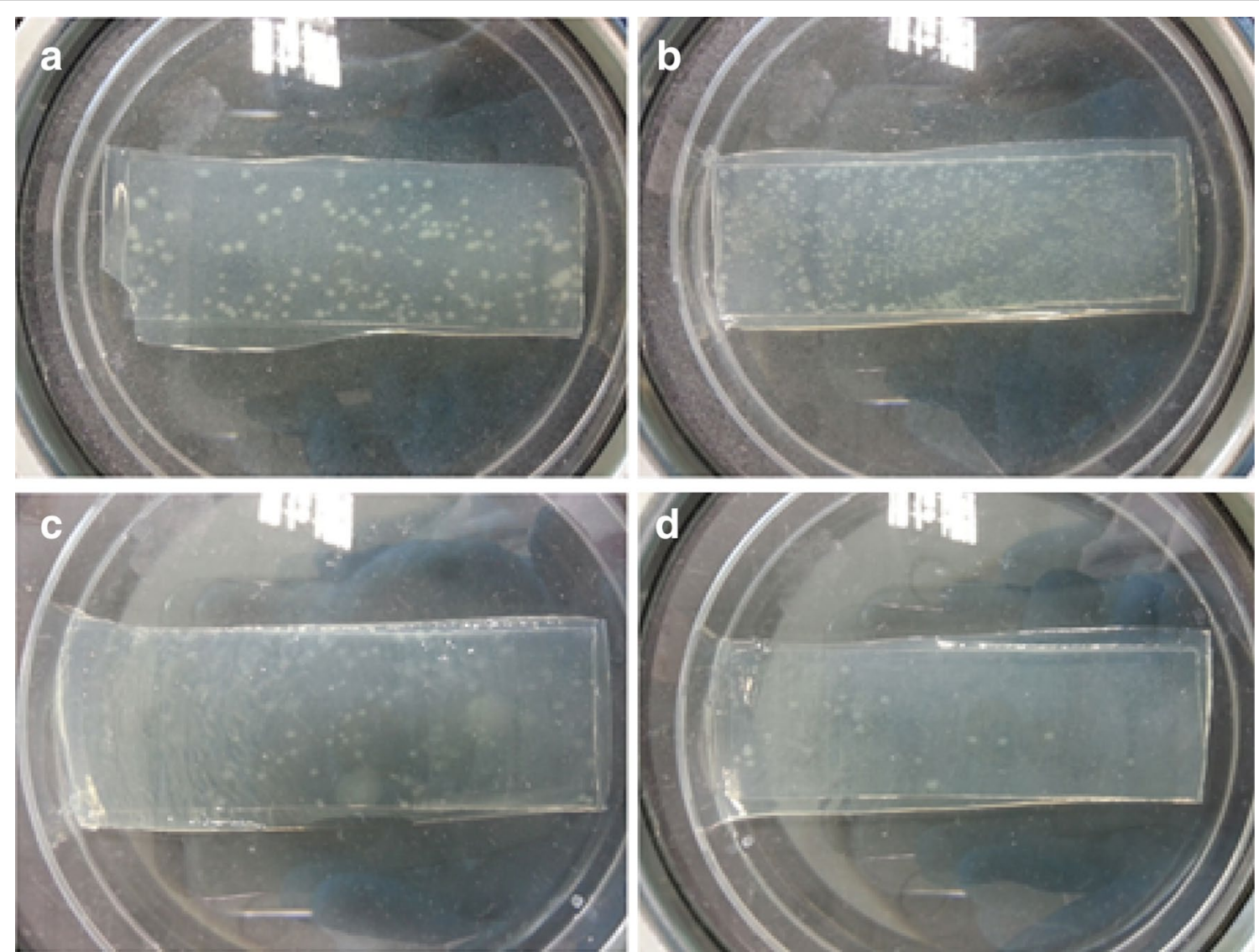

Fig. 3 Effect of organic solvent residues and plasmon treatment time on the antibacterial activity of PDMS films. a Uncleaned and $\mathbf{b}$ cleaned PDMS films before the AgNP fabrication. c Plasma untreated and $\mathbf{d}$ treated PDMS films with AgNP seeds on the film 

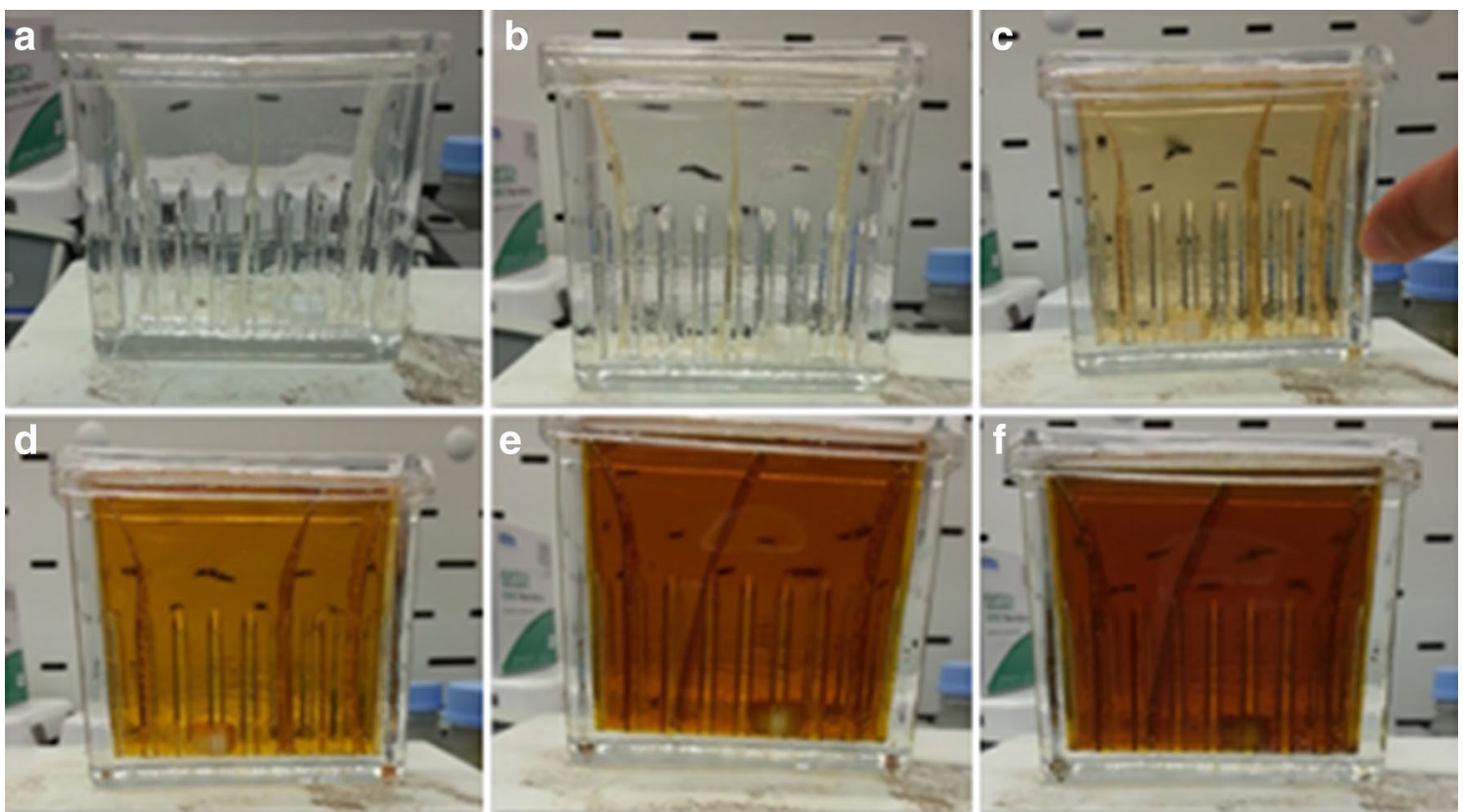

Fig. 4 Time-dependence of the AgNP growing reaction on PDMS film in a solution that contained AgNPs at $80^{\circ} \mathrm{C}$ after the addition of sodium citrate. a $1 \mathrm{~min}, \mathbf{b} 5 \mathrm{~min}, \mathbf{c} 10 \mathrm{~min}, \mathbf{d} 15 \mathrm{~min}$, e $25 \mathrm{~min}$, and $\mathbf{f} 35 \mathrm{~min}$

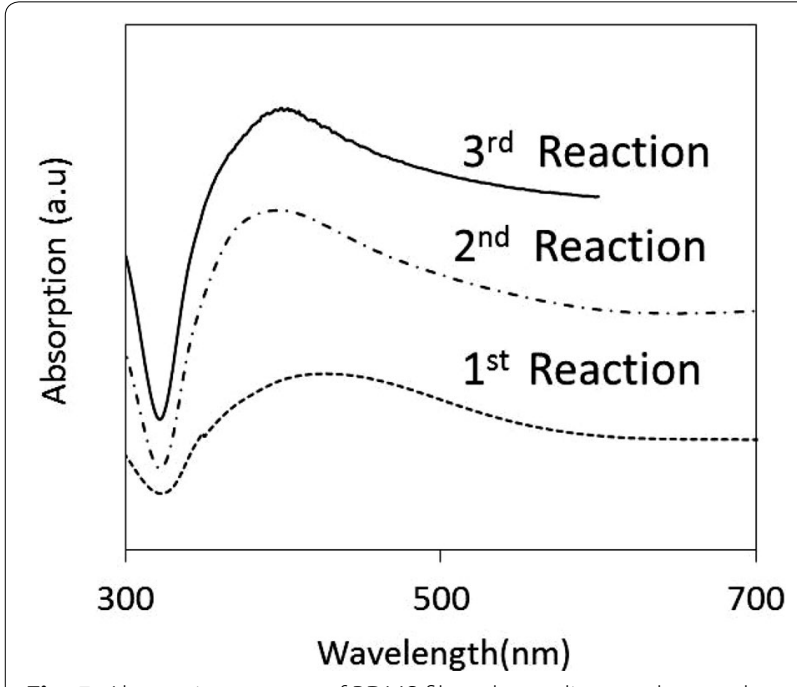

Fig. 5 Absorption spectra of PDMS films depending on the number of rounds of the additional AgNP-growth reaction

on the film. There was a distinct color change of the PDMS film from a colorless transparent surface to a mirror-like surface with either silver or yellow color, depending upon the viewing angle. We carried out this reaction two more times. As the number of reactions increased, the plasmonic peak of the AgNPs increased and the color of the PDMS film darkened. Figure 6 shows scanning microscopic images of PDMS films subjected to different numbers of reactions. The SEM image of the PDS film subjected to one reaction reveals that mostly spherical silver nanostructures had formed. After two or three rounds of reactions, the nanostructures became big and irregular shapes that occupied most of film surface. The matched outline between the SEMS and ion mapping images confirmed the composition of the nanostructure as silver ions. We performed ICP-MS analysis of the AgNP on the film. Of the total weight of film, $0.048 \%$ was accounted for by silver ions.

Before estimating the antibacterial effectiveness, we screened to identify the optimal number of reactions using S. aureus. As shown in Fig. 7 no colony formation was observed on the PDMS films that were reacted for three rounds, suggesting that this treatment resulted in the greatest antibacterial activity.

Therefore, we determined the order of bacterial reduction using the PDMS films subjected to three rounds of reaction based on a method described in ISO 22913. According to this method, the number of colonies after $24 \mathrm{~h}$ incubation with $E$. coli and $S$. aureus that were applied separately to the PDMS film covered with polyethylene film was counted and compared with that on a control film without AgNPs (Fig. 8). The measured bacterial reduction on the PDMS films was $\log _{10} 4.8$ and $\log _{10} 5.7$ for E. coli and S. aureus, respectively. The in situ AgNP grown PDMS films demonstrated greater 

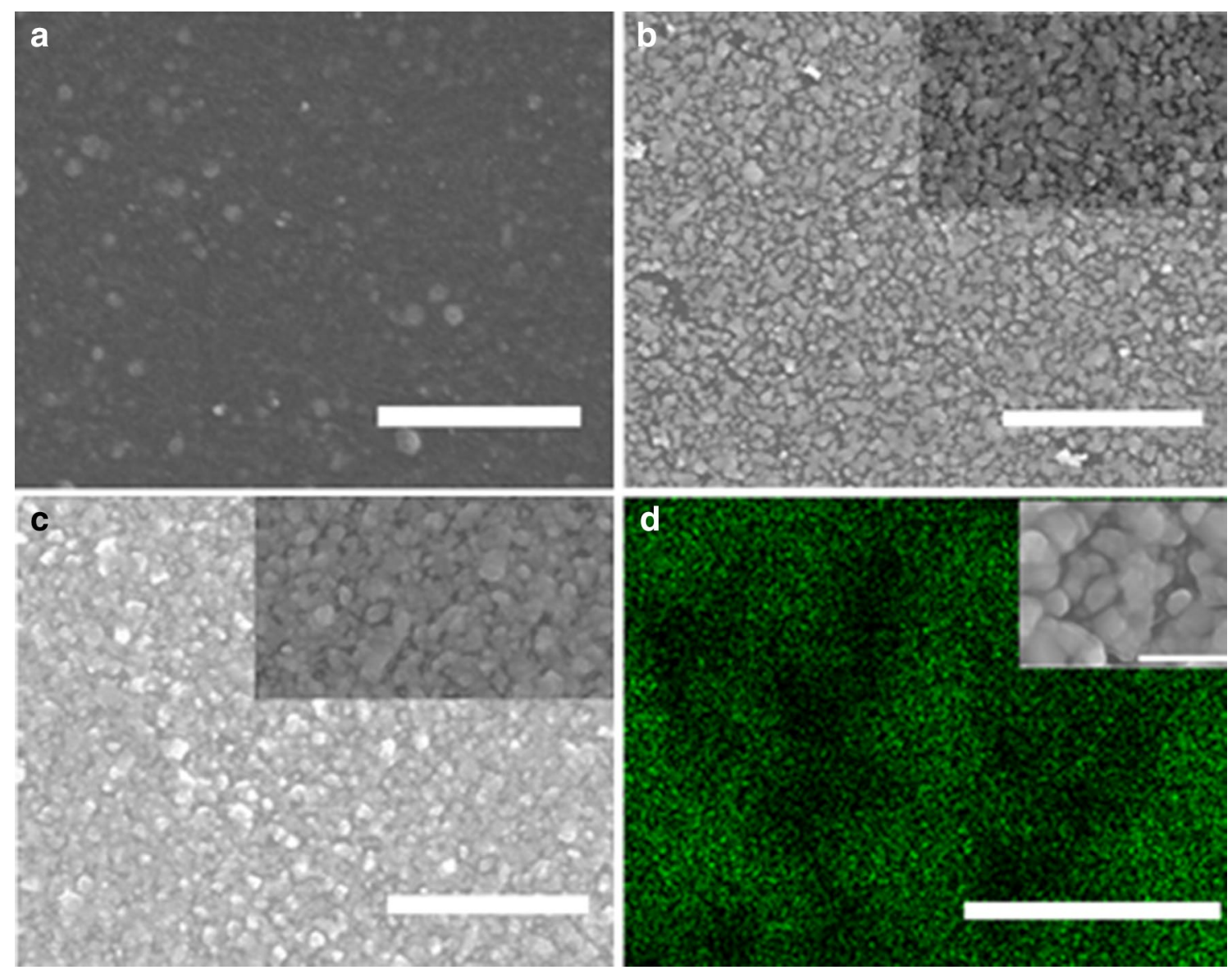

Fig. 6 Electron microscopic analysis of the AgNP-grown PDMS films subjected to various rounds of reactions. From a to c; one, two and three rounds of reaction, respectively. $\mathbf{d}$ lon mapping image and inset of $\mathbf{d}$ corresponding electron microscopic images of AgNP-grown PDMS films after three rounds of the AgNP growing reaction. Insets in $\mathbf{b}$ and $\mathbf{c}$ are enlarged images of the figures. $500 \mathrm{~nm}$ scale bar in each SEM image
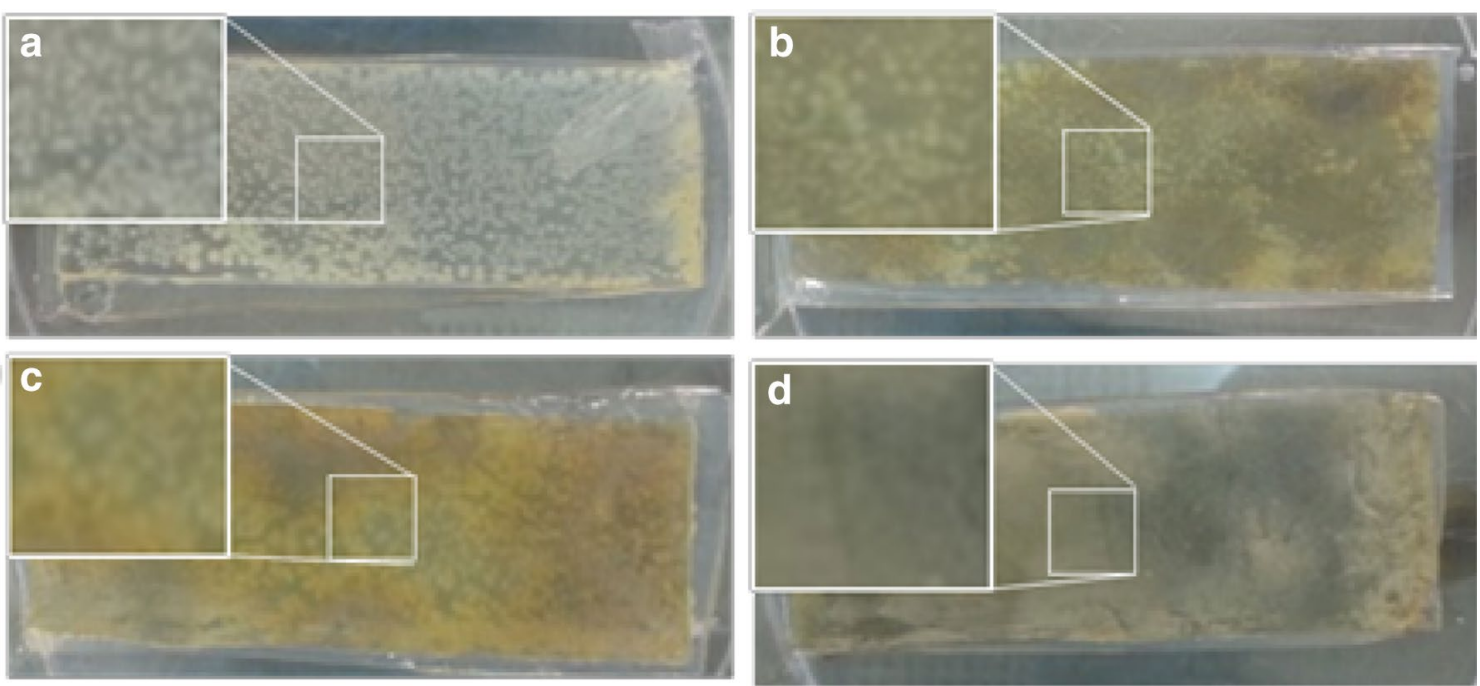

Fig. 7 Photographic images of an AgNP grown PDMS film at $36 \mathrm{~h}$ after inoculation of S. aureus on the surface. a Control PDMS was prepared without the AgNP growing reaction. From $\mathbf{b}$ to $\mathbf{d}$, AgNP-grown PDMS films subjected to one, two, or three rounds of reactions, respectively. Inset in each figure is an enlarged image of the figure 

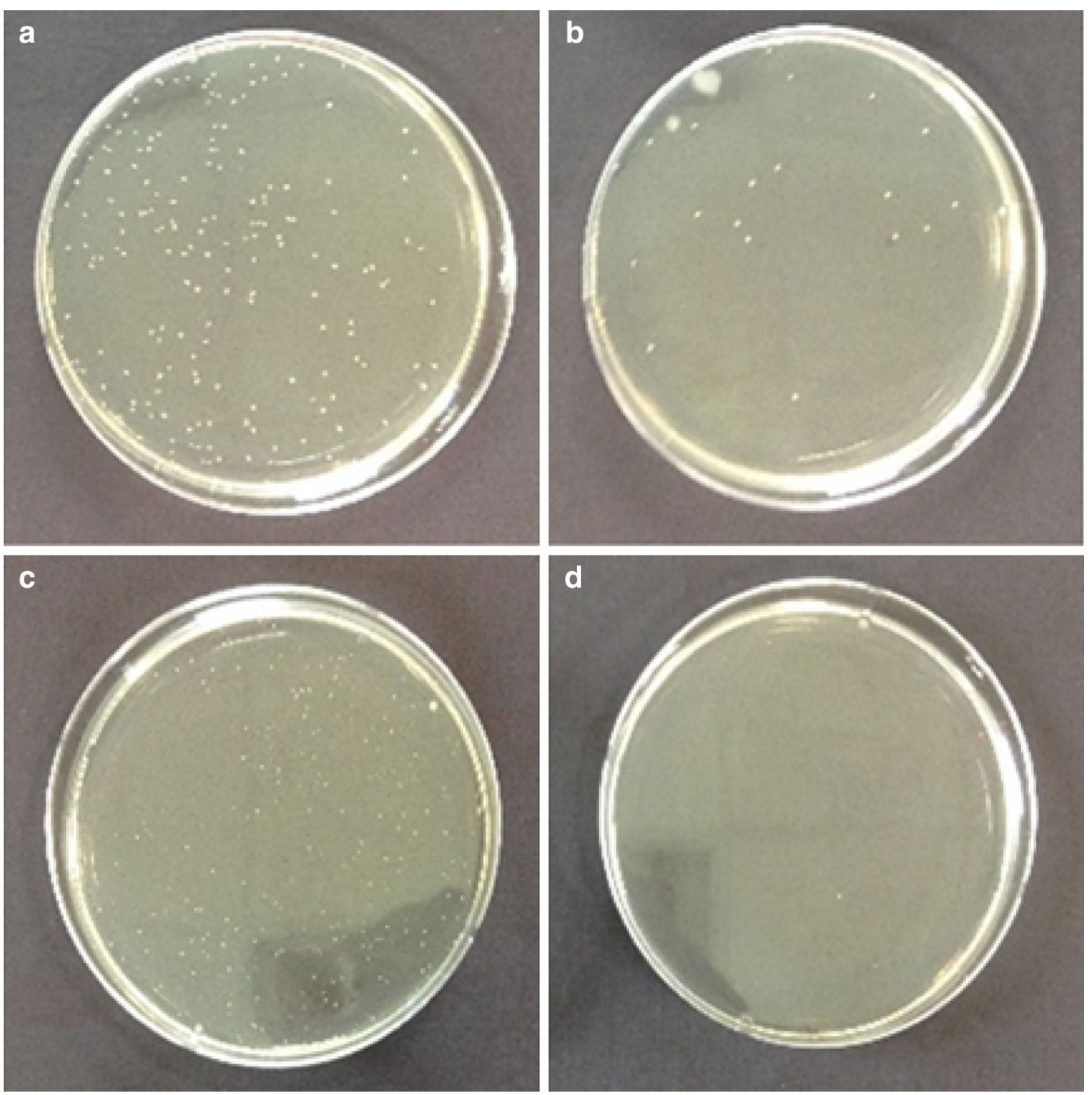

Fig. 8 Colony formations on Mueller-Hinton agar inoculated with diluted solution of the collected elution from the bacterial inoculum on the AgNP-grown PDMS films. a, b E. coli inoculum of $1 \times 10^{5}$ dilution of the control and $1 \times 10^{3}$ dilution of AgNP-grown PDMS film o the agar, respectively. $\mathbf{c}, \mathbf{d}$ S. aureus inoculum of $1 \times 10^{4}$ dilution of the control and $1 \times$ dilution of AgNP-grown PDMS films, respectively

antibacterial activity than the requirement of FDA. Additionally, in order to be used as an implantable medical device, the biological safety of the devices should be guaranteed. We examined the biological safety of the PDMS film by the MTT cytotoxicity test using an eluent of the AgNP-grown PDMS film and direct contact of the films on test cells. As shown in Fig. 9, no significant AgNP-mediated cytotoxicity driven by AgNP on PDMS film was observed. We also examined cell morphology after this treatment. Those cells that contacted the AgNP-grown PDMS films had an identical morphology to the reference cells. These two findings confirmed the biological safety of AgNP-grown PDMS film, as prepared according to the conditions described in this study. Finally, we could fabricate successfully AgNP-attached catheter by applying this in situ method for a preclinical test of the catheters as an antibacterial implantable medical device (Fig. 10). The same color as the AgNP synthesized silicone films indicates formation of AgNP on the treated area of the catheter. Since the catheter was made of silicone rubber powders, the same procedure without any modification as used for the silicon films was applied for the in situ formation of AgNPs on the catheter. 


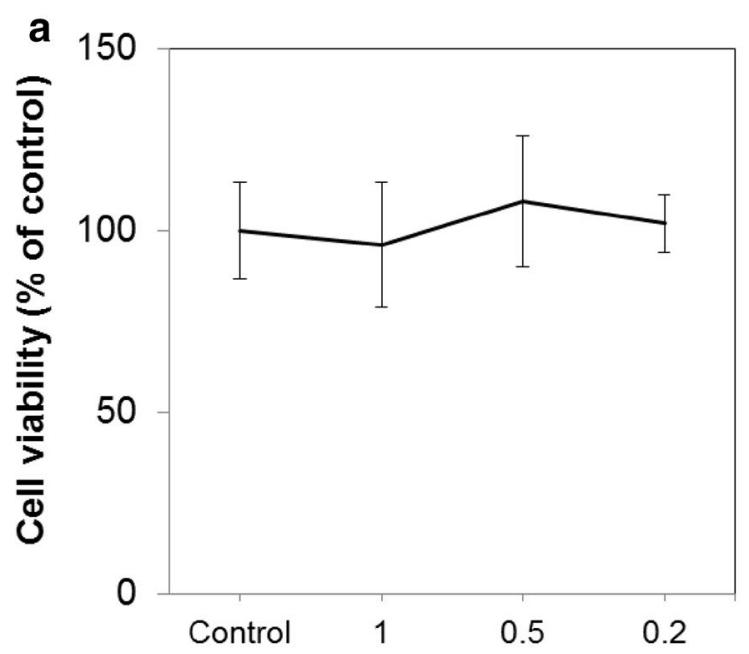

Eluent dilution factor

C

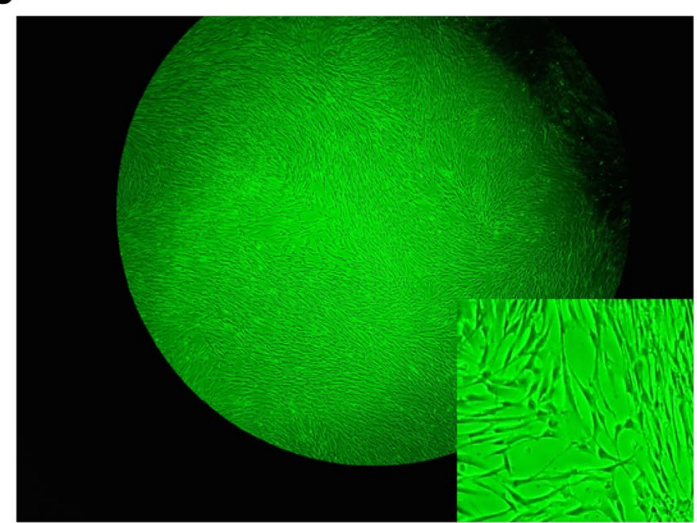

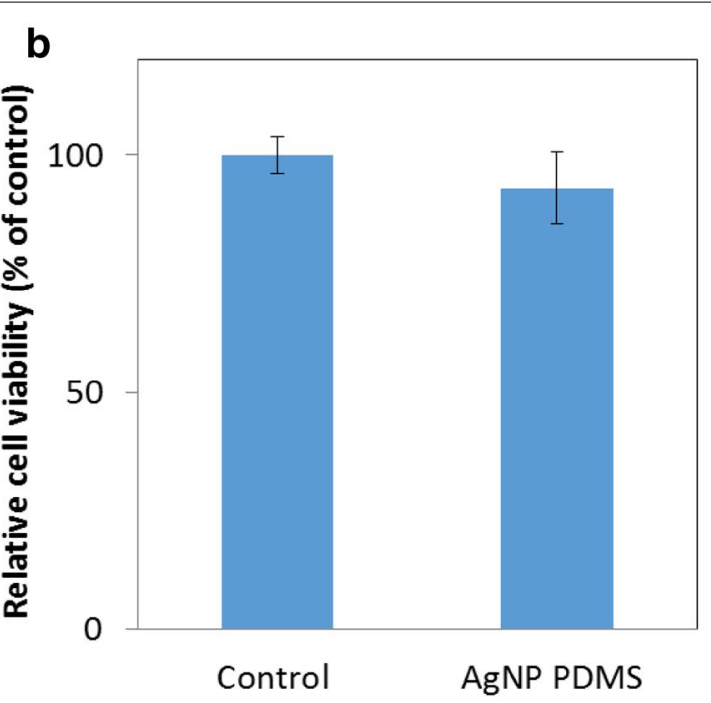

Sample

d

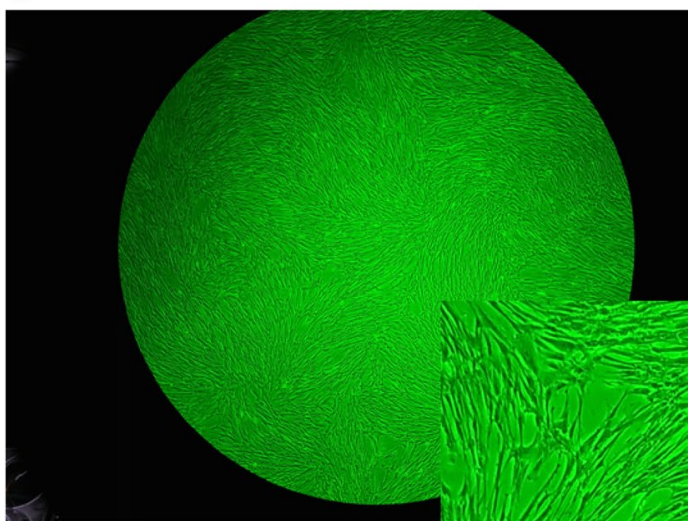

Fig. 9 Cytotoxicity tested with $\mathbf{a}$ eluent and $\mathbf{b}$ direct contact of the AgNP-grown PDMS film. Microscopic images of the tested cells grown in direct contact with c control PDMS film and $\mathbf{d}$ AgNP-grown PDMS films. Inset of $\mathbf{c}$ and $\mathbf{d}$ is enlarged image of each figure

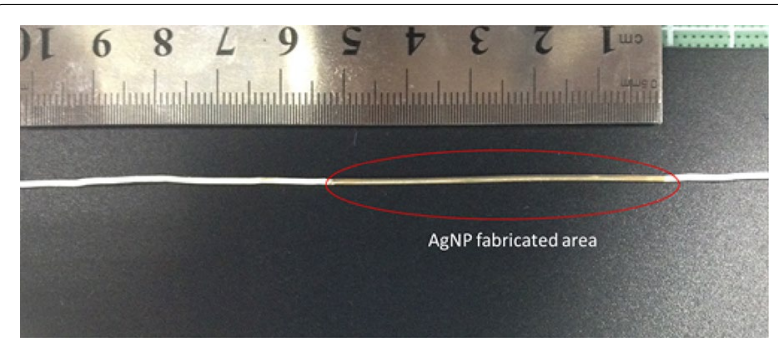

Fig. 10 Photographic image of an in situ AgNP fabricated catheter for use in a pre-clinical test

\section{Conclusion}

In the present study, we successfully demonstrated the in situ synthesis of AgNPs on the PDMS film for use as an antibacterial surface. The AgNP-PDMS composite showed high antibacterial activity against representative infectious bacterial growth. Of the total weight of the film, a small amount was represented by silver. Since all of the AgNPs were placed on the surface of PDMS film, they ( only $0.048 \%$ of the total weigh of the PDMS film) could act to prevent bacterial growth, resulting in effective antibacterial activity. The oxidative release of $\mathrm{Ag}^{+}$ from $\mathrm{Ag}^{0} \mathrm{NP}$ is known to drive antibacterial actions, such as binding to a wide variety of targets that contain organic amines, phosphates, thiol-containing proteins, and DNA, and can increase reactive oxygen species and simultaneously perturb many aspects of the cell metabolism [6]. Thus, the release of silver ions can negatively impact the surrounding cells via similar antibacterial actions. Therefore, it is crucial to minimize the cytotoxicity of the antibacterial surface while maintaining its bacterial killing 
activity. There are several ways to avoid the side effects of AgNPs for uses in medical applications, such as the layer-by-layer technique [22] and plasma immersion ion implantation [23], to prepare AgNP-containing antibacterial polymer films. In a comparison of these methods, the in situ synthesis of AgNPs on the PDMS film presented herein is straightforward and less dependent on instruments. Consequently, it could be easily integrated in the existing manufacturing process of PDMS-based medical devices. Goyal et al. reported an in situ synthesis of AgNP inside of the PDMS film by mixing silver precursors with elastomers and then reducing them [13]. Since most AgNPs were embedded in the film, only 80 and 52\% reduction growth of $E$. coli and $B$. subtilis, respectively, was reported. By contrast, we achieved more than a five $\log _{10}$ reduction of bacteria with a small amount of AgNPs because all AgNPs synthesized in present study were located on the film surface. We believe that our finding could be useful for manufacturing a silicone-based implanting device with antibacterial activity by proving the straightforward and price economically competitive method.

\section{Authors' contributions}

All authors have contributed to the writing of the manuscript. All authors read and approved the final manuscript.

\section{Competing interests}

The authors declare that they have no competing interests.

\section{Funding}

This study was supported by a Grant of the Daegu-Gyeongbuk Medical Innovation Foundation Medical Device Development Center R\&D Project (DG13D001).

\section{Publisher's Note}

Springer Nature remains neutral with regard to jurisdictional claims in published maps and institutional affiliations.
Received: 7 September 2017 Accepted: 3 November 2017

Published online: 27 November 2017

\section{References}

1. H.E. Pearse, Ann. Surg. 124, 1020 (1946)

2. A.J. Domb, Khan W (eds.), Focal Controlled Drug Delivery (Springer, Boston, MA, 2014)

3. A. Colas, J Curtis, in Silicone Biomaterials: History and Chemistry \& Medical Applications of Silicones, 2nd edn, ed. By B.D. Ratner, A.S. Hoffman, F.J. Schoen, J.E. Lemons (Elsevier, Amsterdam, 2004)

4. D.G. Maki, D.M. Kluger, C.J. Crnich, Mayo Clin. Proc. 81, 1159 (2006)

5. M. Chen, Q. Yu, H. Sun, Int. J. Mol. Sci. 14, 18488 (2013)

6. B. Le Ouay, F. Stellacci, Nano Today 10, 339 (2015)

7. P.A. Gunatillake, D.J. Martin, G.F. Meijs, S.J. McCarthy, R. Adhikari, Aus. J. Chem. 56, 545 (2003)

8. Z.M. Davoudi, A.E. Kandjani, A.I. Bhatt, I.L. Kyratzis, A.P. O'Mullane, V. Bansal, Adv. Funct. Mater. 24, 1047 (2014)

9. M.D. Willcox, E.B. Hume, A.K. Vijay, R. Petcavich, J. Optom. 3, 143 (2010)

10. C. Tieszer, G. Reid, J. Denstedt, J. Biomed. Mater. Res. A. 43, 321 (1998)

11. S. Huda, S.K. Smoukov, H. Nakanishi, B. Kowalczyk, K. Bishop, B.A. Grzybowski, A.C.S. Appl, Mater. Interfaces 2, 1206 (2010)

12. S.N. Sawant, V. Selvaraj, V. Prabhawathi, M. Doble, PLoS ONE 8, e63311 (2013)

13. A. Goyal, A. Kumar, P.K. Patra, S. Mahendra, S. Tabatabaei, P.J. Alvarez, G. John, P.M. Ajayan, Macromol. Rapid Comm. 30, 1116 (2009)

14. J. Haldar, A.K. Weight, A.M. Klibanov, Nat. Protoc. 2, 2412 (2007)

15. ISO 22196:2007, Plastics-Measurement of antibacterial activity on plastics surfaces, 1st edn, International Standard (2007)

16. ISO 10993-5, Biological evaluation of medical devices-Part 5: Tests for in vitro cytotoxicity, 3rd edn, International Standard (2009)

17. J.F. Gomes, A.C. Garcia, E.B. Ferreira, C. Pires, V.L. Oliveira, G. Tremiliosi-Filho, L.H. Gasparotto, Phys. Chem. Chem. Phys. 17, 21683 (2015)

18. Q. Bao, D. Zhang, P. Qi, J. Colloid Interf. Sci. 360, 463 (2011)

19. P. Zhang, Y. Huang, X. Lu, S. Zhang, J. Li, G. Wei, Z. Su, Langmuir 30, 8980 (2014)

20. S.K. Sia, G.M. Whitesides, Electrophoresis 24, 3563 (2003)

21. B.T. Ginn, O. Steinbock, Langmuir 19,8117 (2003)

22. A. Agarwal, K.M. Guthrie, C.J. Czuprynski, M.J. Schurr, J.F. McAnulty, C.J. Murphy, N.L. Abbott, Adv. Funct. Mater. 21, 1863 (2011)

23. W. Zhang, Y. Luo, H. Wang, J. Jiang, S. Pu, P.K. Chu, Acta Biomater. 4, 2028 (2008)

\section{Submit your manuscript to a SpringerOpen ${ }^{\circ}$ journal and benefit from:}

- Convenient online submission

- Rigorous peer review

- Open access: articles freely available online

- High visibility within the field

- Retaining the copyright to your article

Submit your next manuscript at springeropen.com 